\title{
Exploring New Options for Data Interpolation with Radial Basis Functions
}

\author{
Reinhard Piltner \\ Email: rpiltner@georgiasouthern.edu \\ Department of Mathematical Sciences, \\ Georgia Southern University, \\ Statesboro, GA 30460, USA
}

\begin{abstract}
In many disciplines there is a need for efficient in terpolation of ir regular spaced data. For unsampled locations values have to be computed from the available data. Usually we are interested in smooth interpolations, and artificial o scillations should be avoided. For large data sets fully populated matrices are undesirable. The use of compactly supported basis functions appears to be attractive. In this paper radial basis functions satisfying the $2 \mathrm{D}$ bi-harmonic equation are used. Accurate interpolation could be achieved in the numerical test examples.
\end{abstract}

Keywords: Data interpolation; Trefftz Method; Radial B asis Functions.

\section{Introduction}

Often data is collected at irregular spaced locations. Computational tools for a fast interpolation at unsampled locations are needed. One huge application area is in the processing of geographic data sets. Many authors made contributions to the topic of data interpolation (see for example $[1,2,4][3]$ )

\section{Problem Statement and Methods}

We consider a 2D domain for which at $\mathrm{N}$ scattered points data values are given. Using radial basis functions we want to find an interpolation function which can recover the data values at the given locations and which can smoothly interpolate between the sampled locations $\left(x_{j}, y_{j}\right)$. When using globally supported radial basis functions (RBFs) we obtain a fully populated coefficient matrix in the linear system of eq uations. Some popular globally supported RBFs are

$$
\begin{aligned}
& \Phi(\mathrm{r})=\sqrt{r^{2}+c^{2}} \\
& \Phi(\mathrm{r})=r^{2} \log r \\
& \Phi(\mathrm{r})=r^{2 n} \log r \\
& \Phi(\mathrm{r})=e^{-c r^{2}}
\end{aligned}
$$

where for the $2 \mathrm{D}$ case

$$
r=\sqrt{\left(x-x_{j}\right)^{2}+\left(y-y_{j}\right)^{2}}
$$

In order to obtain a sparse coefficient matrix in the li near sy stem of equations we can utilize compactly supported radial basis functions. In the following some popular compactly supported RBFs with $C^{n}$ continuity are listed:

$$
\begin{array}{lll}
\Phi(r)=(1-r)_{+}^{2}, & C^{0} \\
\Phi(r)=(1-r)_{+}^{4}(4 r+1), & C^{2} \\
\Phi(r)=(1-r)_{+}^{6}\left(35 r^{2}+18 r+3\right), & C^{4} \\
\Phi(r)=(1-r)_{+}^{8}\left(32 r^{3}+25 r^{2}+8 r+1\right), & & C^{6}
\end{array}
$$




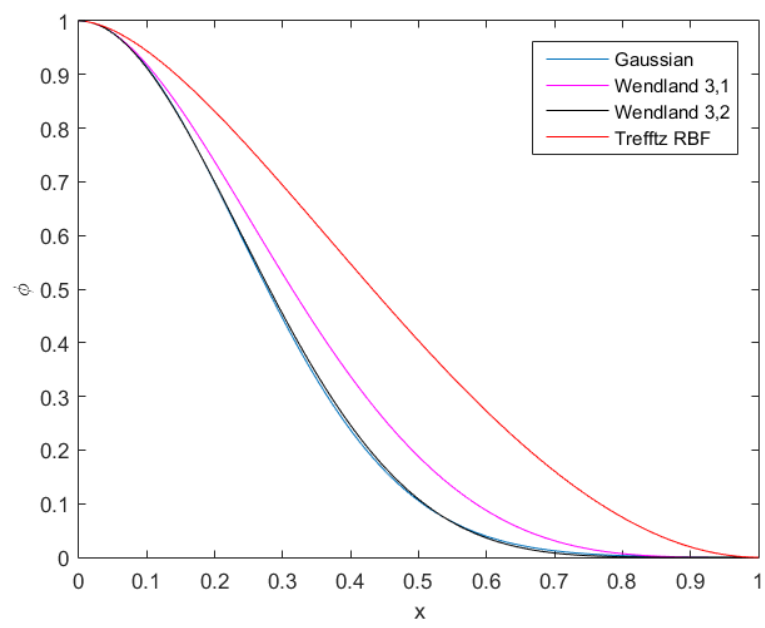

Figure 1: Comparison of a few radial basis functions

In this paper a compactly supported radial basis function with a very special feature is uesed. The RBF has to satisfy the bi-harmonic equation

$$
\Delta \Delta \Phi=0
$$

Solutions of the bi-harmonic equation are expected to be very smooth and very suitable for data interpolation. In the engineering literature one can find solutions of the bi-harmonic equation for rectangular and circular plates subjected to different loading and support conditions. The solution for a clamped circular plate with radius $=a$ loaded at the center is given on page 69 of the book by Timoshenko/Woinowsky-Krieger [5] :

$$
w(r)=\frac{P a^{2}}{16 \pi D}\left[2\left(\frac{r}{a}\right)^{2} \ln \left(\frac{r}{a}\right)+\left(1-\left(\frac{r}{a}\right)^{2}\right)\right]
$$

Utilizing this plate bending solution we can define the following bi-harmonic Trefftz radial basis function:

$$
\Phi(R)=2 R^{2} \ln (R)+\left(1-R^{2}\right)
$$

where $R=\frac{r}{a}$. In the literature often functions satisfying a governing differential equation are called Trefftz trial functions [6] The Trefftz radial basis function can be compared to other radial basis functions in Figure 1 and in Figure 2

\section{$3 \quad$ Numerical Experiments}

In order to test the numerical performance of the Trefftz radial basis functions satisfying the bi-harmonic equation artificial data has been constructed from $t$ widely used Franke test function (Fig. 3). Franke's test function is made up from four exponential function terms with powers of $\mathrm{x}$ and $\mathrm{y}$ in the exponents. At random locations $(\mathrm{x}, \mathrm{y})$ the $\mathrm{z}$-values are computed from Franke's function. The computed z-values are used as the data for the interpolation tests. After solving a linear sparse system of equations for the coefficients of the Trefftz radial basis functions the obtained interpolation function is evaluated at the the original $(\mathrm{x}, \mathrm{y})$ locations. It is seen in Figure 4 that the data values are recovered very well in the algorithm. 


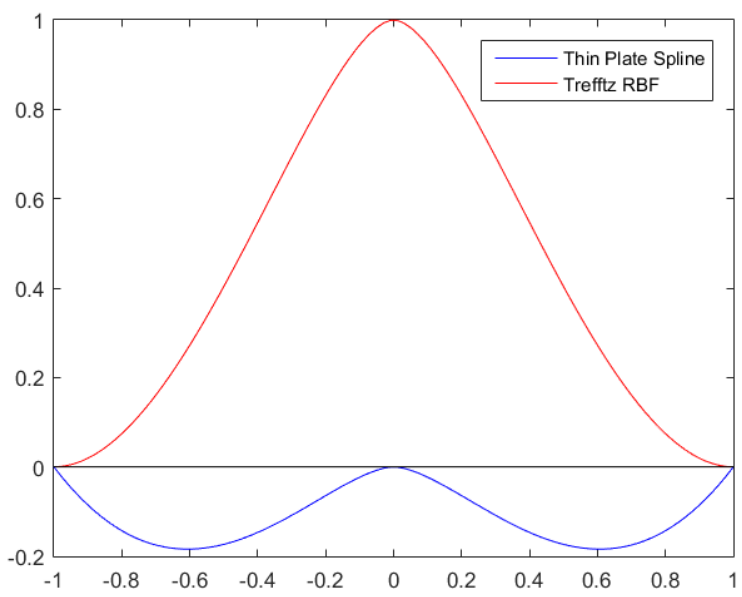

Figure 2: Comparison of a Trefftz radial basis function and a thin plate spline.

\section{Conclusion}

Several globally supported and compactly supported radial basis functions are available from the literature. Globally supported functions are useful for smaller data interpolation tasks. The compactly supported radial basis functions for this paper have the special feature that a bi-harmonic differential equation is satisfied so that the approximation function can be considered a Trefftz function. For a sequence of numerical test problems the chosen Trefftz radial basis function appeared to be well performing. In the near future numerical tests for radial basis functions satisfying homogeneous tri-harmonic equations and satisfying an inhomogeneous bi-harmonic equation will be performed.

\section{References}

[1] Martin D Buhmann. Radial basis functions: theory and implementations. Cambridge Monographs on Applied and Computational Mathematics. Cambridge University Press, Cambridge, 2003.

[2] Peter Lancaster and Kestutis Salkauskas. Curve and surface fitting: an introduction. Academic Press, London, 1986.

[3] T. Losser, L. Li, and R. Piltner. A spatiotemporal interpolation method using radial basis functions for geospatiotemporal big data. In COM.geo, pages 17-24, 2014.

[4] Robert Schaback and Holger Wendland. Characterization and construction of radial basis functions. In Multivariate Approximation and Applications, pages 1-24. Cambridge University Press, 2001.

[5] S. Timoshenko and S. Woinowsky-Krieger. Theory of Plates and Shells. McGraw-Hill, New York, 1959.

[6] E. Trefftz. Ein Gegenstueck zum Ritzschen Verfahren. In 2. Int. Kongr. f. Techn. Mechanik, pages 131-137, Zuerich, 1926. 


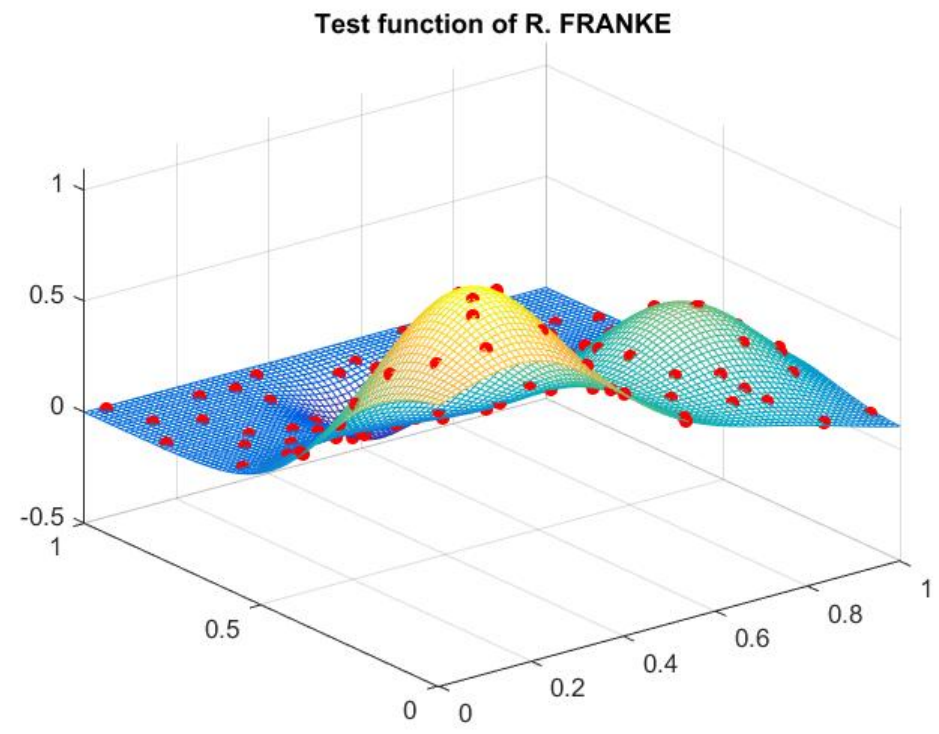

Figure 3: Franke's test function with selected data locations

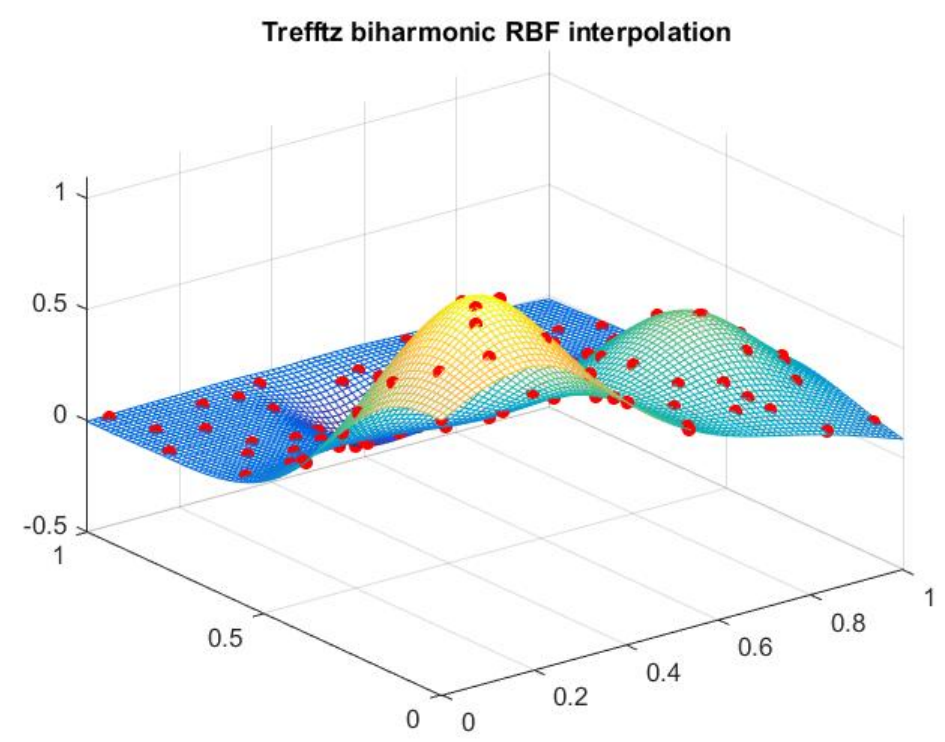

Figure 4: Recovered data values using the Trefftz RBF interpolation. 